\title{
14. A Theorem concerning the Dynamical Systems with Slow Variation.
}

\author{
By Yusuke HagiHaRA. \\ Astronomical Observatory, Azabu, Tokyo. \\ (Comm. by K. Hirayama, M.I.A., Feb. 12, 1931.)
}

1. In certain dynamical systems such as often appear in celestial mechanics the solution is not always convergent ${ }^{1)}$ if it is expressed in series of a form for practical use. Although its convergence in series of a certain form is actually proved in the way of the theory of analytic functions, ${ }^{2)}$ the solution in convergent series can not reveal its nature from the dynamical point of view. Thus we have to employ semi-convergent series in order to get the information as to the behaviour of the motion. It is proposed to estimate the duration of time in which the solution can be approximated by an adopted representation to a previously given degree. A first step toward attacking this problem was trodden by Birkhof ${ }^{3)}$ for a motion near an equilibrium point in which the characteristic numbers are all distinct, different from zero and not connected by any linear homogeneous relation with rational coefficients. The problem of this note ${ }^{4)}$ is to extend the research to the case in which a certain number of characteristic numbers are zero at least in the first approximation.

2. Consider a function $H$ of $2 m+2 n$ variables $x_{i}, y_{i}, \xi_{j}, \eta_{j}$ and $t(i=1,2, \ldots \ldots, m ; j=1,2, \ldots \ldots, n)$, which, together with its partial derivatives of the first order with respect to any of the variables, satisfy the Lipschitz condition for all the variables $\xi_{j}$, $\eta_{j}$ and $t$ and for all values of $\xi_{j}, \eta_{j}$ and $t$ in a domain : $\left|x_{i}\right|,\left|y_{i}\right|<D,(i=1,2, \ldots \ldots, m)$ with a finite positive constant $D$. With this function $H$ associate the following system of differential equations :

1) H. Poincaré: Acta Math. 13 (1889), 1; Méthodes Nouvelles de la Mécanique Céleste. T. 2 (1893).

2) K. Sundman: Acta Math. 36 (1912), 105; T. Levi-Civita: Acta Math. 42 (1918), 99.

3) G. D. Birkhoff : Amer. Jour. Math. 49 (1927), 1; Dynamical Systems. (1927) Chap. IV.

4) It is a great pleasure to express my indebtedness to Professor G. D. Birkhoff for his valuable criticisms and suggestions and to the Rockfellor Foundation for enabling me to work at the Harvard University. 
No. 2.] A Theorem concerning the Dynamical Systems with Slow Variation.

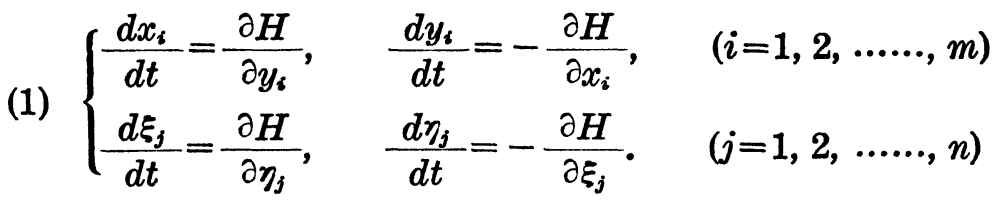

Assume that we have a solution $x_{i}=y_{i}=0, \xi_{j}=A_{j}, r_{j}=B_{j}(i=1,2, \ldots \ldots, m$; $j=1,2, \ldots \ldots, n)$ for all values of $t$, where $A_{j}$ and $B_{j}$ are arbitrary constants.

From this assumption we infer that $H$ can be expressed in the domain $D$ of $x_{i}$ and $y_{i}$ and for all values of $\xi_{j}, \eta_{j}$ and $t$ in a convergent form :

$$
H=\sum_{k, l}^{1,2, \ldots ., m}\left(a_{k l} x_{k} x_{l}+b_{k l} x_{k} y_{l}+c_{k l} y_{k} y_{l}\right)+H_{1}\left(x_{i}, y_{i}, \xi_{j}, \gamma_{j}, t\right),
$$

where $a_{k i l}=a_{l k}, c_{k l}=c_{l k}$ and $H_{1}$ is a power series of $x_{i}$ and $y_{i}$ beginning with the terms of the third degree in $x_{i}$ and $y_{i}$, the coefficients of which are Lipschitzian functions of all the variables $\xi_{j}, \eta_{j}$ and $t$ for all values of $\xi_{j}, \eta_{j}$ and $t$. This is the case in which more than two characteristic numbers are zero in the first approximation. We propose to study the nature of the solution of (1) in $D$.

Assume further that $a_{k l}, b_{k l}, c_{k l}$ are all constants, that the characteristic equation of the quadratic terms of $H$ has only linear invariant factors, that the characteristic numbers are all purely imaginary, and finally that there is no linear homogeneous relation with rational coefficients among the characteristic numbers.

By a linear canonical transformation the system (1) can be transformed into a system for pairs of conjugate imaginary variables $x_{i}^{\prime}$ and $y_{i}^{\prime}$ with an associated system for real pairs $\xi_{j}$ and $\gamma_{j}$ :

$$
\text { (2) }\left\{\begin{array}{cl}
\frac{d x_{i}{ }^{\prime}}{d t}=\frac{\partial F}{\partial y_{i}^{\prime}}, & \frac{d y_{i}^{\prime}}{d t}=-\frac{\partial F}{\partial x_{i}^{\prime}}, \quad(i=1,2, \ldots \ldots, m) \\
\frac{d \xi_{j}}{d t}=\frac{\partial F}{\partial \gamma_{j}}, & \frac{d \eta_{j}}{d t}=-\frac{\partial F}{\partial \xi_{j}}, \quad(j=1,2, \ldots \ldots, n) \\
F={ }^{1,2, \ldots \ldots, m} \lambda_{k} \lambda_{k} x_{k}^{\prime} y_{k}^{\prime}+F_{3}+F_{4}+\cdots \cdots,
\end{array}\right.
$$

where $F_{3}, F_{4}, \ldots \ldots$, are respectively the terms of the third, fourth, ....., degree in $x_{i}^{\prime}$ and $y_{i}^{\prime}$, of which the coefficients are functions of $\xi_{j}, \eta_{j}$ and $t$. $F$ is also convergent. $\lambda_{k}$ 's are the characteristic numbers.

3. Next apply a transformation ;

$$
\bar{x}_{i}=\frac{\partial G}{\partial \bar{y}_{i}}, \quad y_{i}^{\prime}=\frac{\partial G}{\partial x_{i}{ }^{\prime}}, \quad(i=1,2, \ldots \ldots, m)
$$


with

$$
G=\sum_{k}^{1,2, \ldots \cdots,{ }^{m}} x_{k}^{\prime} \bar{y}_{k}+G_{3}+G_{4}+\cdots \cdots+G_{u}+G^{(u)} .
$$

$G_{3}, G_{4}, \ldots \ldots, G_{u}$ are homogeneous functions of $\bar{y}_{i}$ and $x_{i}^{\prime}$ of degree indicated by the suffixes and $G^{(u)}$ is the remainder term as yet undetermined but does not contain terms of the degree $1,2, \ldots \ldots, u$ in $\bar{y}_{i}$ and $x_{i}^{\prime}$. By the $s-2$ times' repetition of this transformation for $u=3,4, \ldots \ldots, s$ we can bring $F$ in the form:

$$
\begin{aligned}
& K=K^{(s)}+R^{(s)}, \quad R^{(s)}=F_{s+1}+F_{s+2}+\cdots \cdots,
\end{aligned}
$$

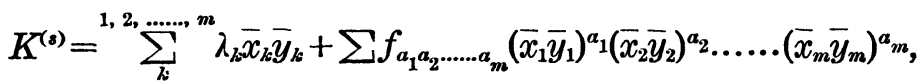

where the last sum extends to all integral values, positive or zero, of $a_{1}, a_{2}, \ldots \ldots, a_{m}$ satisfying

$$
\begin{array}{ll}
2<2\left(a_{1}+a_{2}+\cdots \cdots+a_{m}\right) \leqq s, & \text { if } s \text { is even, } \\
2<2\left(a_{1}+a_{2}+\cdots \cdots+a_{m}\right) \leqq s-1, & \text { if } s \text { is odd, }
\end{array}
$$

and $f_{a_{1} a_{2} \ldots \ldots a_{m}}$ 's are functions of $\xi_{j}, \eta_{j}$ and $t$. And the system (2) is transformed into:

$$
\text { (3) } \begin{cases}\frac{d \bar{x}_{i}}{d t}=\frac{\partial K^{(s)}}{\partial \bar{y}_{i}}+\frac{\partial R^{(s)}}{\partial \bar{y}_{i}}, & \frac{d \bar{y}_{i}}{d t}=-\frac{\partial K^{(s)}}{\partial \bar{x}_{i}}-\frac{\partial R^{(s)}}{\partial \bar{x}_{i}}, \\ \frac{d \overline{\bar{\xi}}_{j}}{d t}=\frac{\partial K^{(s)}}{\partial \bar{\eta}_{j}}+\frac{\partial R^{(s)}}{\partial \bar{\eta}_{j}}, & \frac{d \bar{\eta}_{j}}{d t}=-\frac{\partial K^{(s)}}{\partial \bar{\xi}_{j}}-\frac{\partial R^{(s)}}{\partial \overline{\bar{\xi}}_{j}}, \\ (j=1,2, \ldots, \ldots, n)\end{cases}
$$

where we have replaced $\xi_{j}$ and $\eta_{j}$ by $\bar{\xi}_{j}$ and $\bar{\eta}_{j}$ in order to make the comparison with (1) more comprehensible.

Denote the result of substituting a system of arbitrary constants $c_{i}$ for $\left(\bar{x}_{i} \bar{y}_{i}\right)(i=1,2, \ldots . ., m)$ in $K^{(s)}$ by a parenthesis. Consider a curtate system :

$$
\text { (4) }\left\{\begin{array}{lll}
\frac{d \bar{x}_{i}}{d t}=\left(\lambda_{i}+\frac{\partial K^{\prime}}{\partial c_{i}}\right) \bar{x}_{i}, & \frac{d \bar{y}_{i}}{d t}=-\left(\lambda_{i}+\frac{\partial K^{\prime}}{\partial c_{i}}\right) \bar{y}_{i}, & (i=1,2, \ldots \ldots, m) \\
\frac{d \bar{\xi}_{j}}{d t}=\left(\frac{\partial K^{\prime}}{\partial \bar{\eta}_{j}}\right), & \frac{d \bar{\eta}_{j}}{d t}=-\left(\frac{\partial K^{\prime}}{\partial \bar{\xi}_{j}}\right), & (j=1,2, \ldots, \ldots, n)
\end{array}\right.
$$

in which $K^{\prime}$ is a finite power series arranged in ascending powers of the constants $c_{1}, c_{2}, \ldots . ., c_{m}$ beginning with the terms of the second degree, the coefficients of the various powers of $c_{i}$ 's being Lipschitzian functions of $\bar{\xi}_{j}, \bar{\eta}_{j}$ and $t$ for all these variables. The system of the form (4) may be said to be normalised. 
Suppose that we can find an exact solution of the normalised associated curtate system :

(5) $\frac{d \bar{\xi}_{i}}{d t}=\left(\frac{\partial K^{\prime}}{\partial \bar{\eta}_{j}}\right), \quad \frac{d \bar{\eta}_{j}}{d t}=-\left(\frac{\partial K^{\prime}}{\partial \bar{\xi}_{j}}\right) . \quad(j=1,2, \ldots \ldots, n)$

Denote by $x_{i}(t), y_{i}(t), \xi_{j}(t), \eta_{j}(t)$ the solution of the system of differential equations (1) with a certain initial condition with the restriction that $x_{i} y_{i}=c_{i}$ for $t=t_{0}$. Let $\bar{x}_{i}(t), \bar{y}_{i}(t), \bar{\xi}_{j}(t), \bar{\eta}_{j}(t)$ be the corresponding solution of the system (4) with the same initial condition and the same restriction. Then the relations of the form:

$$
\begin{aligned}
\left|x_{i}(t)-\bar{x}_{i}(t)\right|,\left|y_{i}(t)-\bar{y}_{i}(t)\right|, & \left|\xi_{j}(t)-\bar{\xi}_{j}(t)\right|,\left|\gamma_{j}(t)-\bar{\eta}_{j}(t)\right|<\delta, \\
& (i=1,2, \ldots \ldots, m ; \quad j=1,2, \ldots \ldots, n)
\end{aligned}
$$

hold with a previously assigned positive number $\delta$ in the time interval :

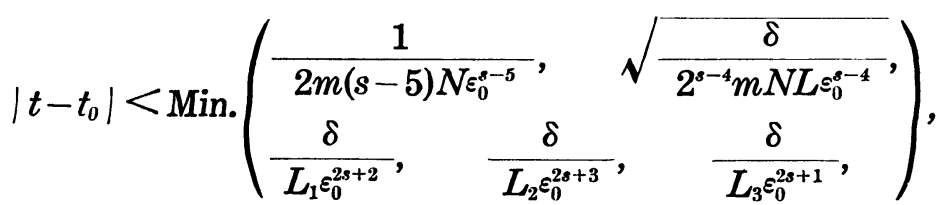

where $\varepsilon_{0}$ is the value of $\varepsilon=\left(\sum_{k}^{1,2}, \ldots,{ }^{m} \bar{x}_{k} \bar{y}_{k}\right)^{\frac{1}{2}}$ for $t=t_{0}$ and $L, L_{1}, L_{2}, L_{3}$ and $N$ are finite positive constants depending on $\varepsilon_{0}, c_{i}, s$ and on the functional form of $F$ but neither vanishing nor becoming infinite when $\varepsilon_{0}$ tends to zero. Or, if $\delta=O\left(\varepsilon_{0}^{\alpha}\right)$ in Landau's notation, then

$$
\left|x_{i}(t)-\bar{x}_{i}(t)\right|,\left|y_{i}(t)-\bar{y}_{i}(t)\right|,\left|\xi_{j}(t)-\bar{\xi}_{j}(t)\right|,\left|\eta_{j}(t)-\bar{\eta}_{j}(t)\right|=O\left(\varepsilon_{0}^{x}\right),
$$

in the time interval contained between the limits extending on both sides of $t_{0}$ :

or

$$
\begin{array}{lll} 
\pm\left|t-t_{0}\right|=O\left(\varepsilon_{0}^{\frac{x-s+4}{2}}\right) & \text { for } & x>-s+6, \\
\pm\left|t-t_{0}\right|=O\left(\varepsilon_{0}^{-s+5}\right) & \text { for } & x<-s+6 .
\end{array}
$$

4. Especially if the system (5) is satisfied by quasi-periodic functions of Bohl and Esclangon, ${ }^{1}$ then the solution of (1) can be approximated by a stable motion expressed by quasi-periodic functions in the domain just mentioned to the degree of approximation $\delta$.

If (5) admits the point $\xi_{j}=A_{j}, \eta_{j}=B_{j},(j=1,2, \ldots \ldots, n)$ as a centre $e^{2)}$ in the sense of Poincaré, then (1) is also approximated by a stable solution. Vranceanu's case ${ }^{3)}$ is a further specification of this corollary.

1) P. Bohl : Dorpat. Dissertation (1893); Crelle J. 131 (1906), 268; E. Esclangon : Paris. Thèse (1904) ; Ann. Obs. Bordeaux (1904); C. R. 135 (1902), 891 ; 137 (1903), 305; H. Bohr : Acta Math. 46 (1925), 101.

2) H. Poincaré: Jour. de Math. [iv] 1 (1885), 167; Oeuvres. T. 1.

3) C. Vranceanu: Atti Accademia Lincei. Rendiconti. [vi] 7 (1928), 630. 